\title{
Overexpression of the MUC1 Gene in Iranian Women with Breast Cancer Micrometastasis
}

\author{
Neda Mansouri ${ }^{1}$, Abolfazl Movafagh ${ }^{1 *}$, Shahrzad Soleimani ${ }^{1}$, Mohammad \\ Taheri $^{1}$, Mehrdad Hashemi ${ }^{2}$, Atefeh Heidary Pour ${ }^{3}$, Shohreh Alizadeh Shargh ${ }^{4}$, \\ Alireza Mosavi-Jarahi ${ }^{5}$, Zahra Sasaninejad ${ }^{6}$, Hanieh Zham $^{7}$, Parastoo Hajian, \\ Hossein Allah Moradi ${ }^{8}$, Hamid Reza Mirzaei ${ }^{7}$, Hedieh Fardmanesh ${ }^{9}$, Mina \\ Ohadi $^{10}$
}

\begin{abstract}
The membrane epithelial mucin MUC1 is expressed at the luminal surface of most simple epithelial cells, but expression is greatly increased in most breast cancers. The aims of present study were to investigate expression of the MUC1 gene and interactive affects in metastases. Whole cell RNA isolation from 50 sentinel lymph nodes (SNLs) of breast cancer patients was performed using reverse transcription and real-time PCR. All patients were diagnosed with breast cancer and without metastasis, confirmed by IHC staining. The evaluation of tumor and normal samples for expression of MUC1 gene, the results were $49.1 \%$ non-expressive and $45.3 \%$ expression (Student $\mathrm{t}, \mathrm{p}=0.03$ ). Also in comparison of normal breast tissue and breast cancer SLN for MUC1 gene, MUC1 negative SLNs were $75.0 \%$ (18 samples) and MUC1 positive samples were 25.0\% (6 samples). Over-expression of MUC1 gene may offer a target for therapy related to progression and metastasis in women with breast cancer.
\end{abstract}

Keywords: Breast cancer- micrometastasis- sentinel lymph node- real-time PCR

Asian Pac J Cancer Prev, 17, Cancer Control in Western Asia Special Issue, 275-278

\section{Introduction}

Evaluation of the sentinel lymph nodes (SLNs) for the presence of cancer is an important part of breast cancer staging. Detection of micrometastasis in SLNs in patients with invasive breast cancer can help prevent risk of recurrence in comparison with those whose lymph nodes are completely free of cancer, and therefore early identification of clinically relevant disease could lead to new treatment or staging approaches for breast cancer.

Mucin1 (MUC1) is a transmembrane glycoprotein normally expressed at low levels on the apical surface of epithelial cells. In most adenocarcinomas and in other inflammatory diseases of epithelial tissues, MUC1 is overexpressed and loses the polarity of its expression. The large extracellular domain contains the variable number of tandem repeats region (VNTR) composed of around 20-200 tandem repeat (TR) units of 20 amino acids each, and is rich in prolines and
O-glycosylated serines and threonines (Suzuki et al., 2004). The extracellular domain is non-covalently linked to the smaller subunit consisting of an autocatalytic cleavage SEA-module, the transmembrane domain (TM) and the cytoplasmic tail (CT).

On normal epithelia, the MUC1 VNTR is extensively O-glycosylated with long branched glycans, whereas on tumor cells the VNTR is markedly hypoglycosylated with simpler and shorter glycan chains. Elevated levels of MUC1 on the tumor have been associated with invasiveness and poor prognosis in colon, pancreas, breast and bladder cancer (Hollingsworth., 2004). There is additional evidence that MUC1 contributes to the growth and metastatic properties of tumors (Kamata et al., 2002; Schroeder et al., 2003; Tinder et al., 2008). The cytoplasmic tail of MUC1 (MUC1,CT) was reported to be involved in a wide range of intracellular signaling processes by associating with several protein partners and modulating their function. Recently we showed that the extracellular domain and the VNTR also participate in

\footnotetext{
${ }^{1}$ Department of Medical Genetics, ${ }^{5}$ Department of Epidemiology, School of Medicine, ${ }^{7}$ Cancer Research Center, Shohadae Tajrish Hospital, Department of Radiation Oncology, ${ }^{8}$ Department of General Medicen, Shahid Beheshti University of Medical Sciences, ${ }^{2}$ Department of Genetics, Tehran medical sciences branch, ${ }^{3}$ Department of Cell and Molecular Biology, Pharmaceutical Sciences Branch, ${ }^{6}$ Department of genetic, Islamic Azad University, ${ }^{10}$ Iranian Research Center on Aging, University of Social Welfare and Rehabilitation Sciences, Tehran, ${ }^{4}$ Medical Laboratory Science Department, Midwifery-Nursing Institute, Islamic Azad University of Chalous Branch, Chalous, ${ }^{9}$ Molecular Medicine Research Center, Hormozgan University of Medical Sciences, Bandar Abbas, Iran. *For correspondence: movafagh_a@yahoo.com
} 
intracellular signaling by activating the NF- $\mathrm{kB}$ pathway (Cascio et al., 2011). In addition to higher levels of MUC1 in tumors compared to normal cells, hypoglycosylation of the VNTR is the most important characteristic of tumor MUC1. Fewer sugars on the VNTR increase the accessibility of the peptide backbone to more efficient or completely new protein-protein interactions that can profoundly change intracellular signaling in tumors compared to normal cells (Fontenot et al., 2008; TaylorPapadimitriou et al., 2002). We investigated the proteins that associate with MUC1 and their interactive affect in tumor cells.

\section{Material and Methods}

Fifty consecutive patients with breast carcinoma underwent SLN biopsy dissection at the Shohada Tajrish referral and teaching Hospital affiliated to Shahid Beheshti University of Medical Sciences (Tehran, Iran) from Jun 2014 to Nov 2015. All patients were diagnosed with breast cancer and without metastasis, confirmed by IHC staining. The study design and patients were assigned on the basis of national/international breast cancer protocols and approved according to the local terms and regulations, by the Institutional Review Boards of each participating referral hospital. The study was performed at Shahid Beheshti University of Medical Sciences, in adherence to the guidelines of the Declaration of Helsinki, and was approved by the ethics committee of Shahid Beheshti University of Medical Sciences Tehran, Iran.

\section{Total RNA Isolation and cDNA Synthesis}

Whole cell RNA isolation from SNLs of breast cancer patient were performed using and homogenized in Tripure reagent, nearly $50 \mu \mathrm{g}$ of SLN specimen was added to 1 $\mathrm{mL}$ of Tripure and homogenized. Then incubation from 5 minutes at room temperature and then added $0.2 \mathrm{ml}$ of chloroform and homogenized and centrifuged in 12,000 for 15 minutes at $4^{\circ} \mathrm{C}$. RNA was precipitated retained and added isopropyl alcohol. Upper aqueous phase was removed. Then centrifuged in 12,000 for 10 minutes at $4^{\circ} \mathrm{C}$, and then pellet was rinsed twice $1 \mathrm{~mL}$ of ethanol $(75 \%)$. The RNA was re suspended in DEPS water to a concentration of $0.5 \mu \mathrm{g} / \mu \mathrm{l}$.

\section{Reverse Transcription and Real-time PCR}

$0.5 \mu \mathrm{g}$ of oligo dT and $16 \mu \mathrm{L}$ RNase free water were added to $5 \mu \mathrm{g}$ of whole RNA and incubated at $64^{\circ} \mathrm{C}$ for 10 minute. Total RNA was extracted. The primers for SYBR Green real-time PCR were designed specificly for each checkpoint gene, and for ACTB gene ( $\beta$-actin) as internal control. The assays were repeated for each measurement.

Reverse Transcription was carried out with the Super Script First-Strand Synthesis System for RT-PCR. The procedure was based on Invitrogen's protocol (total RNA $5 \mu \mathrm{g}$, random hexamers $(50 \mathrm{ng} / \mu \mathrm{l}) 3 \mu \mathrm{l}, 10 \mathrm{mM}$ dNTP mix $1 \mu \mathrm{l}$, DEPC H2O to $10 \mu \mathrm{l})$. The samples were incubate at $65^{\circ} \mathrm{C}$ for $5 \mathrm{~min}$ and then on ice for at least $1 \mathrm{~min}$. Master mix was made as follows: 10x RT buffer $2 \mu \mathrm{l}, 25 \mathrm{mM}$ $\mathrm{MgCl} 24 \mu \mathrm{l}, 0.1 \mathrm{M}$ DTT $2 \mu \mathrm{l}$, RNAase OUT $1 \mu \mathrm{l}$. The reaction mixture was added to the RNA/primer mixture and mixed briefly, and then placeed at room temperature for 2 min. One $\mu 1$ (50 units) of Super Script II RT was added to each tube, mixed and incubated at $25^{\circ} \mathrm{C}$ for 10 $\min$. The tubes were incubated at $42^{\circ} \mathrm{C}$ for 50 min, heat inactivated at $70^{\circ} \mathrm{C}$ for $15 \mathrm{~min}$, and then chilled on ice. One $\mu \mathrm{l}$ RNase $\mathrm{H}$ was added and incubated at $37^{\circ} \mathrm{C}$ for 20 min, and the 1 st strand cDNA was stored at $-20^{\circ} \mathrm{C}$ until use for Real-time PCR.

MUC1(forward):5' GTATAAAACGGAAGCAGCC TCTC3', MUC1(reverse):5'GAGAGGCTGCTTCCGT TTTATAC3,

ACTB(Reverse): 5'TGATTTTCCCAGCGAGTTCT3' ACTB(forward):5'CCAGGCACCACGAAGTGAT GG3'

\section{Results}

The RT-PCR reaction was performed on all cancer and tumor samples as described earlier and the raw data were analyzed with SPSS 14 software with t-test and Pearson's chi-square analysis (Figure 1). Also, the specificity of RT-PCR products was determined. All RT-PCR products

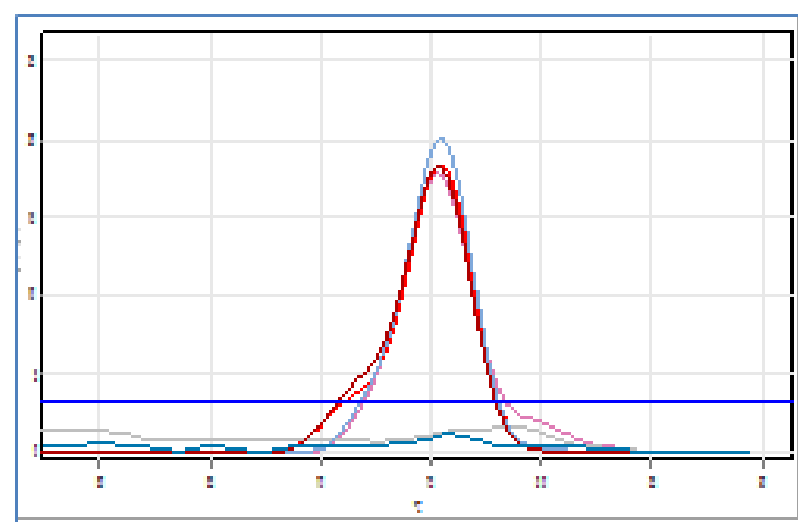

Figure 1. The Melting Curve of RT-PCR Analysis for MUC1 Gene, Tm: $81^{\circ} \mathrm{C}$.

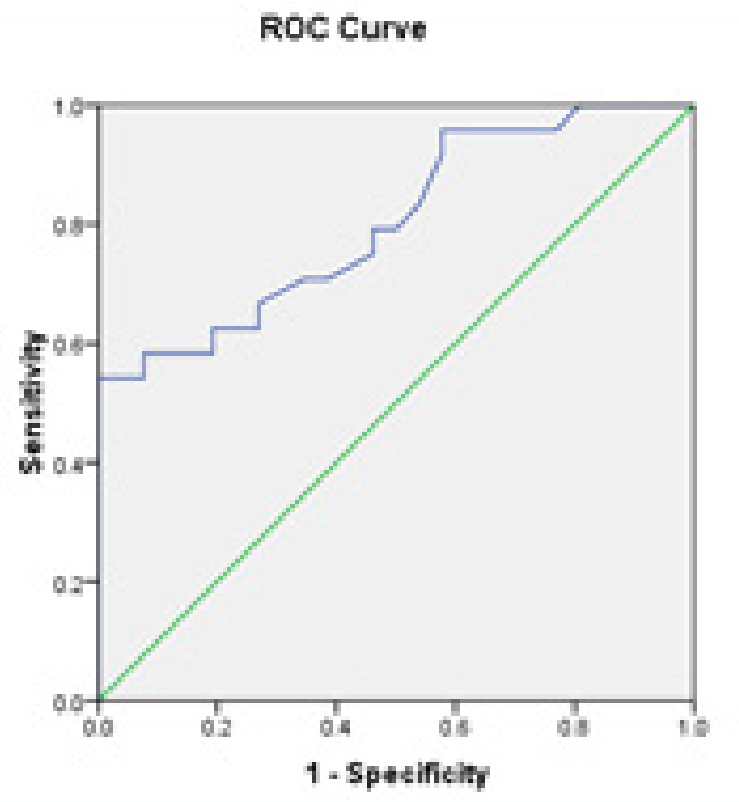

Figure 2 : ROC Analysis for Test Accuracy for MUC1 Gene, Area Under Curve 0.8 Case Processing Summary, Positive $=24$, Negative $=26$, Missing $=3$ 
Table 1. Comparison of Sentinel Lymph Node and Normal Breast Tissue for MUC1 Gene Expression

\begin{tabular}{|c|c|c|c|c|c|c|c|c|c|c|}
\hline \multicolumn{11}{|c|}{ Paired Samples Test } \\
\hline & & \multicolumn{3}{|c|}{ Paired Differences } & & & & \multirow[t]{2}{*}{$\mathrm{t}$} & \multirow[t]{2}{*}{$\mathrm{df}$} & \multirow{2}{*}{$\begin{array}{l}\text { Sig. } \\
\text { (2-tailed) }\end{array}$} \\
\hline & & Mean & $\begin{array}{l}\text { Std. } \\
\text { Deviation }\end{array}$ & Std. Mean & Error & $\begin{array}{l}95 \% \mathrm{C} \\
\text { the Dif }\end{array}$ & erval of & & & \\
\hline & & & & & & Lower & Upper & & & \\
\hline \multirow[t]{2}{*}{ Pair 1} & $\begin{array}{l}\text { muc1EXP. } \\
\text { Cancer }\end{array}$ & & 2.4 & 0.3 & & -1.4 & -.08 & & 49.0 & 0.03 \\
\hline & $\begin{array}{l}\text { muc1EXP. } \\
\text { Normal }\end{array}$ & -0.8 & & & & & & -2.2 & & \\
\hline
\end{tabular}

were electerophoresed in $2 \%$ agaroge gel for specifying the products.

In evaluation of tumor and normal samples for expression of MUC1 gene, there was $49.1 \%$ non-expressive and $45.3 \%$ expression ( $\mathrm{t}$-student $\mathrm{p}=0.03$ ) (Table 1 ).

Also in comparison of normal breast tissue and breast cancer SLN for MUC1 gene, MUC1 negative SLNs were $75.0 \%$ ( 18 sample) and MUC1 positive samples were $25.0 \%$ (6 sample), were also ER negative and from 43.3\% (11 sample) ER negative and 57.7\% (15 sample) ER positive, were MUC1 negative, that the significant difference was seen $(\mathrm{p}<0.01)$.

In evaluation of MUC1 expression and tumor's stage, there was no significant correlation $(p=0.11)$ but with grade, there was a meaningful difference $(p=0.03)$, means that with higher grade, the expression of MUC1 is reduced. The results of ROC analysis for MUC1 gene is shown as follows (Figure 2).

\section{Discussion}

Cancer is a heterogeneous and complex disease that has different pathological and cytological features. Cytogenetic and molecular diagnoses are important challenges for prognosis and treatment of this disease. MUC1 has been proposed to be a key modulator of several signaling pathways in epithelial cancers that affect tumor cell invasion, proliferation and survival (Kimura et al., 200; Besmer et al., 2011). The above is supported by the importance of this gene as a diagnostic marker of poor prognosis and as a target for therapeutic intervention in cancer. The contribution of MUC1 to the invasive and metastatic properties of adenocarcinomas has been linked with some forms of MUC1 increasing the adhesive properties of tumor cells, and others promoting anti-adhesiveness (e.g. interacting with ICAM-1 and galectin-3), as well as by regulating cell signaling. MUC1 associates with $\beta$-catenin, NF- $\kappa B$, and EGFR, and translocates to the nucleus, where it regulates transcription of several genes responsible for progression and invasiveness of cancer (Bernier et al., 2011; Roy et al., 2010). Previous studies indicate that CIN85 is a binding partner of MUC1, and implicate the MUC1/CIN85 complex in several of these functions. CIN85 is an adaptor protein involved in cellular processes such as ubiquitination, endocytic internalization and intracellular trafficking. CIN85 is highly expressed in human squamous cell carcinoma of the head and neck and in cervical carcinoma, and this overexpression is significantly correlated with advanced clinical stages of disease (Wakasaki et al., 2010; Ma et al., 2010).

Molecular markers such HOXB13 make the prognosis much accurate and can predict the metastasis potential of cancer (Nishidate et al., 2004). Studies on paraffin embedded breast cancer samples have shown the relation between mammaglobin gene and metastatic outcome of cancer (Wang et al,. 2010). In another study using RT-PCR on mamaglobin on blood sample and cancer tissue biopsy, the relation between low grade (I,II) with ER positive samples has been reported (Ouellette et al., 2004). In evaluation of MUC1 and CK19 expression in pathologic negative SLNs, it has been found that the level of at least one of these genes is elevated. However, because of false positivity of MUC1 in normal samples, this gene had been excluded from further studies (Saintigny et al., 2005). Studies showed that MUC1 is destroyed via ubiqutination in ER positive breast cancers, but the exact underlying mechanism is not well known (Wei et al., 2006). Our results revealed that the expression of MUC1 has significant variation between normal and tumor samples $(p=0.03)$. It seems that MUC1 acts through activation of NF-KB pathway and induction of mammosphere production for progression of malignant cells. Also, there was meaningful relation with tumor grade $(p=0.03)$ but not with tumor's stage $(\mathrm{p}=0.11)$, and with the ER status $(p=0.02)$ (the latter has been reported previously in ER positive patients) (Shargh SA et al., 2014). Although further analyses are required, it is possible that CIN85 is responsible for MUC1 expression at the apical plasma membrane in normal cells and in early stages of tumors, and for its expression on the entire surface and in the cytosol in advanced stages of tumors. Remarkably, MUC1 and CIN85 co-localize at invadopodia-like protrusions (Pegoraro et al., 2013). As the invasive capacity of cancer cells depends, in part, on their ability to assemble invadopodia, the formation of the MUC1/ CIN85 complex may be a requirement for this process (Colditz GA et al., 2014). In this study, we show for the first time, overexpression of MUC1 in Iranian women with breast cancer that can be related to early cancer development, progression, and metastasis. Therapies targeting MUC1 over-expression may be considered in Iranian women with breast cancer.

\section{Acknowledgment}


This work was supported by deputy research administration, Shahid Beheshti University of Medical Sciences, Tehran, Iran.” grant GR No: -451-20-11-1392. Also the result of this research work is the thesis work of Neda Mansouri. The authors declare no conflict of interest.

\section{References}

Bernier AJ, Zhang J, Lillehoj E, et al (2011). Non-cysteine linked MUC1 cytoplasmic dimers are required for Src recruitment and ICAM-1 binding induced cell invasion. Mol Cancer, 10, 93.

Besmer DM, Curry JM, Roy LD, et al (2011). Pancreatic ductal adenocarcinoma mice lacking mucin 1 have a profound defect in tumor growth and metastasis. Cancer Res, 71, 4432-42.

Cascio S, Zhang L, Finn OJ (2011). MUC1 protein expression in tumor cells regulates transcription of proinflammatory cytokines by forming a complex with nuclear factor-kappaB p65 and binding to cytokine promoters. J Biol Chem, 286, 42248-56.

Colditz GA, Bohlke K, Berkey CS (2014). Breast cancer risk accumulation starts early: prevention must also. Breast Cancer Res Treat, 145, 567-79

Fardmanesh H, Shekari M, Movafagh A, et al (2016). Upregulation of the double-stranded RNA binding protein DGCR8 in invasive ductal breast carcinoma. Gene, $\mathbf{5 8}$, 146-51.

Fontenot JD, Mariappan SV, Catasti P, et al (1995). Structure of a tumor associated antigen containing a tandemly repeated immunodominant epitope. J Biomol Struct Dyn, 13, 245-60.

Heidari MH, Porghasem M, Mirzaei N, et al (2014). The effect of high level natural ionizing radiation on expression of PSA, CA19-9 and CEA tumor markers in blood serum of inhabitants of Ramsar, Iran. J Environ Radioact, 128, 64-7.

Hollingsworth MA, Swanson BJ (2004). Mucins in cancer: protection and control of the cell surface. Nat Rev Cancer, 4, 45-60.

Kamata M, Denda-Nagai K, Kubota N, et al (2002). Vaccination of mice with MUC1 cDNA suppresses the development of lung metastases. Clin Exp Metastasis, 19, 689-96.

Kimura T, Tanaka S, Haruma K, et al (2000). Clinical significance of MUC1 and E-cadherin expression, cellular proliferation, and angiogenesis at the deepest invasive portion of colorectal cancer. Int J Oncol,16, 55-64.

Ma Y, Ye F, Xie X, et al (2010). Significance of PTPRZ1 and CIN85 expression in cervical carcinoma. Arch Gynecol Obstet, 284, 699-704.

Movafagh A, Hajifathali A, Isfahani F, et al (2012). Geographic heterogeneity of cytogenetic characteristics of acute myeloid leukemia in the early detection. Iran J Cancer Prev, 2, 85-9.

Movafagh A, Mirfakhraei R, Mousavi-Jarrahi A (2011). Frequent incidence of double minute chromosomes in cancers, with special up-to-date reference to leukemia. Asian Pac J Cancer Prev, 12, 3453-6.

Movafagh A, Hajifathali A, Zamani M (2011). Secondary chromosomal abnormalities of de novo acute myeloid leukemia-a first report from the Middle East. Asian Pac J Cancer Prev, 12, 2991-4.

Nishidate T, Katagiri T, Lin ML, et al (2004). Genome-wide gene-expression profiles of breast-cancer cells purified with laser microbeam microdissection: identificationof genes associated with progression and metastasis. Int J Oncol, 25, 797-819.

Ouellette RJ, Richard D, Maïcas E (2004). RT-PCR for mammaglobin genes, MGB1 and MGB2, identifies breast cancer micrometastases in sentinel lymph nodes. Am J Clin Pathol, 121, 637-43.

Pegoraro S, Ros G, Piazza S, et al (2013). HMGA1 promotes metastatic processes in basal-like breast cancer regulating EMT and stem ness. Oncotarget, 4, 1293-308.

Roy LD, Sahraei M, Subramani DB, et al (2010). MUC1 enhances invasiveness of pancreatic cancer cells by inducing epithelial to mesenchymal transition. Oncogene, 30,1449-59.

Saintigny P, Coulon S, Kambouchner M, et al (2005). Real-time RT-PCR detection of CK19, CK7 and MUC1 mRNA for diagnosis of lymph node micrometastases in non small cell lung carcinoma. Int $J$ Cancer, 115, 777-82.

Schroeder JA, Adriance MC, Thompson MC, et al (2003). MUC1 alters beta-catenin-dependent tumor formation and promotes cellular invasion. Oncogene, 22, 1324-32.

Seifi-Alan M, Shamsi R, Ghafouri-Fard S, et al (2014). Expression analysis of two cancer-testis genes, FBXO39 and TDRD4, in breast cancer tissues and cell lines. Asian Pac J Cancer Prev, 14, 6625-9.

Shargh SA, Sakizli M, Khalaj V, et al. (2014) Downregulation of E-cadherin expression in breast cancer by promoter hypermethylation and its relation with progression and prognosis of tumor. Med Oncol, 31, 250.

Suzuki H, Shoda J, Kawamoto T, et al (2004). Expression of MUC1 recognized by monoclonal antibody MY.1E12 is a useful biomarker for tumor aggressiveness of advanced colon carcinoma. Clin Exp Metastasis, 21, 321-9.

Taylor-Papadimitriou J, Burchell JM, Plunkett T, et al (2002). MUC1 and the immunobiology of cancer. J Mammary Gland Biol Neoplasia, 7, 209-21.

Tinder TL, Subramani DB, Basu GD, et al (2008). MUC1 enhances tumor progression and contributes toward immunosuppression in a mouse model of spontaneous pancreatic adenocarcinoma. J Immunol, 181, 3116-25.

Wakasaki T, Masuda M, Niiro H, et al (2010). A critical role of c-Cbl-interacting protein of $85 \mathrm{kDa}$ in the development and progression of head and neck squamous cell carcinomas through the ras-ERK pathway. Neoplasia, 12, 789-96.

Wang X, Zachary S, Vierkant RA, et al (2010). Association of genetic variation in mitotic kinases with breast cancer risk. Breast Cancer Res Treat, 119, 453-62.

Wei X, Xu H, Kufe D (2006). MUC1 oncoprotein stabilizes and activates estrogen receptor alpha. Mol Cell, 20, 295-305. 